\title{
ON THE $l$-FUNCTION AND THE REDUCED VOLUME OF PERELMAN II
}

\author{
RUGANG YE
}

\begin{abstract}
In this paper we present a major application of the $l$-function and the reduced volume of Perelman, namely their application to the analysis of the asymptotical limits of $\kappa$-solutions of the Ricci flow.
\end{abstract}

\section{INTRODUCTION}

This paper is a sequel of [Y2]. In [Y2], a number of geometric and analytic properties of the $l$-function and the reduced volume of Perelman were derived. In this sequel, we present a major application of the $l$-function and the reduced volume, namely their application to the analysis of the asymptotical limits of $\kappa$ solutions. Our focus is to present a complete and detailed proof of Proposition 11.2 in $[\mathrm{P} 1]$, which provides asymptotical convergence of $\kappa$-solutions and identifies the asymptotical limits to be nonflat gradient shrinking solitons. A main foundation for the proof is a study of the limit $l$-function and the asymptotical reduced volume (see Definition 1) on the asymptotical limits of $\kappa$-solutions. This is an extension and further development of the theory presented in [Y2].

Recall that $\kappa$-solutions are $\kappa$-noncollapsed nonflat ancient solutions of the Ricci flow with bounded and nonnegative curvature operator. In dimension three, rescaling limits of a solution of the Ricci flow near a blow-up singularity are $\kappa$-solutions. In higher dimensions they also arise as the same kind of rescaling limits under suitable assumptions on the curvature. In other words, in various situations, blow-up singularities of the Ricci flow are modelled on $\kappa$-solutions. For this reason, it is important to understand the structures of $\kappa$-solutions. To this purpose, one performs blow-down rescalings of a given $\kappa$-solution, extracts smooth limits via the process, and identifies them. This is precisely the content of [P1, Proposition 11.2]. This proposition plays an important role in the analysis of blow-up singularities presented in Perelman's papers [P1] and [P2].

Now we give a more detailed account of the content of this paper. In Section 2 , we first derive an upper bound for $\Delta l$ (the Laplacian of the $l$-function) in the weak sense. Then we derive an analytic lemma concerning strong convergence of Sobolev functions. These two tools yield some nice convergence properties of $\kappa$ solutions upon appoaching asymptotical limits, and will be needed for proving [P1, Proposition 11.2], as will be explained below.

Section 3 and Section 4 are devoted to the proof of [P1, Proposition 11.2]. The proof follows the sketch of proof in [P1] for [P1, Proposition 11.2] and incorporates

Received by the editors May 20, 2006 and, in revised form, September 1, 2006.

2000 Mathematics Subject Classification. Primary 53C20, 53C21. 
the ideas in Sections 9 and 10 in [P1] regarding how to identify gradient shrinking solitons. The statements of the theorem and the proof of the convergence part are given in Section 3, while the main part of the identification of the asymptotical limits is presented in Section 4. The proof of the convergence part is based on the estimates in [Y2, Theorem 2.18] (in particular the curvature estimate [Y2, (2.53)] which is derived from [P1, (7.16)] ) and the upper bound for $l$ in [Y2, Lemma 3.2].

The identification of the asymptotical limits can be compared with [Y2, Theorem 4.9], which says that a solution of the backward Ricci flow must be a gradient shrinking soliton, provided that the values of its reduced volume at two different times are equal. Recall that, basically, the said equality forces the weakly formulated differential inequality [Y2, 2.73] (i.e. [P1, (7.13)]) to become an equality, which then leads to the smoothness of the $l$-function and implies that the differential inequality $[\mathrm{Y} 2,(2.74)]$ (i.e. $[\mathrm{P} 1,(7.14)]$ ) also becomes an equality. These two equalities combined with [Y2, Lemma 4.8] (i.e. [P1, Proposition 9.1]) then lead to the desired gradient shrinking soliton conclusion. We apply this strategy to the current situation. First, in Section 3, we apply Perelman's lower bound for $l$ as presented in [Y2] to show that the asymptotical reduced volume on an asymptotical limit has constant values at all times. Next, in Section 4, we apply the upper bound for $\Delta l$ and the convergence lemma in Section 2 to obtain the local $L^{2}$ strong convergence of $\nabla l$ both in space and in spacetime, and use it to derive the differential inequalities $[\mathrm{Y} 2,(2.73)]$ and $[\mathrm{Y} 2,(2.74)]$ in the weak sense for the asymptotical limits. Using this strong convergence we also derive the connection between the two differential inequalitites, namely the fact that one becoming an equality forces the other to become an equality. The remaining steps are similar to the proof of [Y2, Theorem 4.9] in [Y2].

We are grateful to Perelman for providing his lower bound for $l$ as presented in [Y2]. We would also like to thank G. Wei for helpful and stimulating discussions.

\section{Preliminaries}

Consider a smooth solution $g=g(\tau)$ of the backward Ricci flow

$$
\frac{\partial g}{\partial \tau}=2 R i c
$$

on an $n$-dimensional manifold $M$ over an interval $[0, T)$. We assume that $g(\tau)$ is complete for each $\tau \in[0, T)$. Choose an arbitary point $p \in M$ as the $l$-base, i.e. the reference point for the $l$-function; see [Y1, Definition 1].

We'll follow the notations and conventions in [Y1]. In particular, the volume form of a Riemannian metric $h$ on a manifold will be denoted by $d q_{h}$ or simply $d q$. The distance function of $h$ will be denoted by $d_{h}$ or simply $d$.

Lemma 2.1. Assume that the curvature operator is nonnegative on $[0, T)$. Let $\bar{\tau} \in(0, T)$. Then there is a positive constant $C$ depending only on the dimension $n$ and the magnitude of $\frac{\bar{\tau}}{T-\bar{\tau}}$, such that the differential inequlity $\Delta l \leq C \frac{l}{\tau}$ holds true for each $\tau \in(0, \bar{\tau}]$ in the weak sense, i.e.

$$
\int_{M} \nabla l \cdot \nabla \phi d q \geq-\int_{M} C \frac{l}{\tau} \phi d q
$$

for all nonnegative Lipschitz functions $\phi$ with compact support, as well as functions $\phi$ in the Sobolev space $W_{\text {loc }}^{1,2}(N)$ with compact support. (Note that $C$ depends only on $n$ if $T=\infty$.) 
Proof. By [Y2, Theorem 2.23], the differential inequality

$$
\Delta l \leq \frac{|\nabla l|^{2}}{2}+\frac{R}{2}+\frac{l-n}{2 \tau}
$$

holds true in the weak sense. By the estimates in [Y2, Theorem 2.18], on $[0, \bar{\tau}]$ the right hand side of (2.3) is bounded above by $C \frac{l}{\tau}$. The desired conclusion follows.

Next we present an analytic lemma regarding strong convergence of Sobolev functions.

Lemma 2.2. Let $(N, h)$ be a compact Riemannian manifold, where $h$ denotes the metric. Let $f_{k}$ be a bounded sequence of functions in the Sobolev space $W^{1,2}(N)$ such that $f_{k}-f_{k^{\prime}} \in L^{\infty}(N)$ for all $k, k^{\prime}$, and that

$$
f_{k}-f_{k^{\prime}} \rightarrow 0
$$

in $L^{\infty}$ as $k, k^{\prime} \rightarrow \infty$. Furthermore, assume that there is a positive constant $C$ such that $\Delta f_{k} \leq C$ for all $k$ in the weak sense, i.e.

$$
\int_{N} \nabla f_{k} \cdot \nabla \phi d q \geq-C \int_{N} \phi d q
$$

for all nonnegative $\phi \in W^{1,2}(N)$. Then $f_{k}$ converges strongly in $W^{1,2}(N)$ to a function $f$. Consequently, we have

$$
\int_{N}\left|\nabla f_{k}\right|^{2} \eta d q \rightarrow \int_{N}|\nabla f|^{2} \eta d q
$$

for each $\eta \in L^{\infty}(N)$. The same conclusion holds true if $\Delta f_{k} \leq C$ is replaced by $\Delta f_{k} \geq-C$.

Proof. Since $f_{k} \in L^{2}(N),(2.4)$ implies that $f_{k}$ is a Cauchy sequence in $L^{2}(N)$, and hence converges strongly in $L^{2}(N)$ to a function $f$. Then the boundedness of $f_{k}$ in $W^{1,2}(N)$ implies that $f \in W^{1,2}(N)$ and that $f_{k}$ converges weakly to $f$. Furthermore, we have $f_{k}-f \in L^{\infty}(N)$ and $f_{k}-f \rightarrow 0$ in $L^{\infty}(N)$. Taking a limit in $(2.5)$ we deduce that

$$
\int_{N} \nabla f \cdot \nabla \phi d q \geq-C \int_{N} \phi d q
$$

for all nonneagtive $\phi \in W^{1,2}(N)$, i.e. $\Delta f \leq C$ in the weak sense.

We set $\delta_{k}=\sup \left|f_{k}-f\right|$. Then $\delta_{k} \rightarrow 0, f-f_{k}+\delta_{k} \geq 0$ and $f_{k}-f+\delta_{k} \geq 0$. Choosing $\phi=f-f_{k}+\delta_{k}$ in (2.5) we deduce that

$$
\int_{N} \nabla f_{k} \cdot \nabla f d q-\int_{N}\left|\nabla f_{k}\right|^{2} d q \geq-C \int_{N}\left(f-f_{k}+\delta_{k}\right) d q .
$$

Choosing $\phi=f-f_{k}+\delta_{k}$ in (2.7) we deduce that

$$
\int_{N}|\nabla f|^{2} d q-\int_{N} \nabla f \cdot \nabla f_{k} d q \geq-C \int_{N}\left(f-f_{k}+\delta_{k}\right) d q .
$$

It follows that

$$
\int_{N}|\nabla f|^{2} d q-\int_{N}\left|\nabla f_{k}\right|^{2} d q \geq 2 C \int_{N}\left(f_{k}-f-\delta_{k}\right) d q .
$$

On the other hand, choosing $\phi=f_{k}-f+\delta_{k}$ in (2.5) and (2.7) we infer that

$$
\int_{N}|\nabla f|^{2} d q-\int_{N}\left|\nabla f_{k}\right|^{2} d q \leq 2 C \int_{N}\left(f_{k}-f+\delta_{k}\right) d q .
$$


Obviously, (2.10) and (2.11) imply

$$
\int_{N}\left|\nabla f_{k}\right|^{2} d q \rightarrow \int_{N}|\nabla f|^{2} d q
$$

It is easy to see that (2.12) implies that $f_{k}$ converges strongly to $f$ in $W^{1,2}(N)$. Indeed, we have

$$
\int_{N}\left|\nabla f_{k}-\nabla f\right|^{2} d q=\int_{N}\left|\nabla f_{k}\right|^{2} d q+\int_{N}|\nabla f|^{2} d q-2 \int_{N} \nabla f_{k} \cdot \nabla f d q .
$$

The right hand side is easily seen to converge to zero.

Now we derive (2.6). We have

$$
\begin{aligned}
\int_{N}\left|\nabla f_{k}\right|^{2} \eta d q-\int_{N}|\nabla f|^{2} \eta d q= & \int_{N}\left(\nabla f_{k}-\nabla f\right) \cdot \nabla f \eta d q \\
& +\int_{N}\left(\nabla f_{k}-\nabla f\right) \cdot \nabla f_{k} \eta d q .
\end{aligned}
$$

Applying Cauchy-Schwarz inequality and the strong convergence of $\nabla f_{k}$ to $\nabla f$ in $L^{2}$, we then arrive at $(2.6)$.

The proof in the case $\Delta f_{k} \geq-C$ is similar.

It is easy to extend this lemma to the situation of noncompact manifolds. For convenience, we formulate the result in the set-up of pointed manifolds.

Lemma 2.3. Let $(N, h, p)$ be a pointed complete noncompact Riemannian manifold. Let $f_{k}$ be a locally bounded sequence of functions in $W_{l o c}^{1,2}(N)$ such that the $\Delta f_{k}$ are locally unformly bounded from above in the weak sense, namely there exist for each $A>0$ positive constants $C_{1}(A)$ and $C_{2}(A)$ such that for each $k$ the $W^{1,2}$-norm of $f_{k}$ on the geodesic ball $d(p, \cdot)<A$ is bounded above by $C_{1}(A)$, and $\Delta f_{k} \leq C_{2}(A)$ holds true on $d(p, \cdot)<A$ in the weak sense, i.e.

$$
\int_{N_{k}} \nabla f_{k} \cdot \nabla \phi d q \geq-C_{2}(A) \int_{N} \phi d q
$$

for all nonnegative $\phi \in W_{\text {loc }}^{1,2}(N)$ with support contained in $d(p, \cdot)<A$. Furthermore, assume that $f_{k}-f_{k^{\prime}} \in L_{l o c}^{\infty}(N)$ for all $k, k^{\prime}$, and $f_{k}-f_{k^{\prime}} \rightarrow 0$ in $L_{l o c}^{\infty}(N)$. Then $f_{k}$ converges strongly in $W_{l o c}^{1,2}$ to a function $f$. Consequently, we have

$$
\int_{N}\left|\nabla f_{k}\right|^{2} \eta d q \rightarrow \int_{N}|\nabla f|^{2} \eta d q
$$

for each $\eta \in L^{\infty}(N)$ with compact support.

The same conclusion holds true if the $\Delta f_{k}$ are assumed to be locally uniformly bounded from below in the weak sense instead of from above.

Proof. First, $f_{k}$ converges to some $f$ weakly in $W_{l o c}^{1,2}(N)$. Moreover, $f_{k}-f$ converges to zero in $L_{l o c}^{\infty}(N)$. As in the proof of Lemma 2.2 we obtain by taking a limit in

$$
\int_{N} \nabla f \cdot \nabla \phi d q \geq-C_{A} \int_{N} \phi d q
$$

for all $A>0$ and all nonnegative $\phi \in W_{l o c}^{1,2}(N)$ with support contained in $d(p, \cdot)<$ $A$, where $C_{A}=C_{2}(A)$. For a fixed $A>0$, we set $\delta_{k}=\sup _{d(p, \cdot) \leq 2 A}\left|f_{k}-f\right|$. Let $\psi$ be a nonnegative Lipschitz function on $N$ which takes the value 1 on $d(p, \cdot) \leq A$ 
and is zero on $d(p, \cdot) \geq 2 A$. Choosing $\phi=\psi\left(f-f_{k}+\delta_{k}\right)$ in (2.15) and (2.17) we deduce that

$$
\begin{aligned}
\int_{N}|\nabla f|^{2} d q-\int_{N}\left|\nabla f_{k}\right|^{2} d q & \geq 2 C_{2 A} \int_{N} \psi\left(f_{k}-f-\delta_{k}\right) d q+\int_{N} \nabla f_{k} \cdot \nabla \psi\left(f_{k}-f-\delta_{k}\right) d q \\
& +\int_{N} \nabla f \cdot \nabla \psi\left(f_{k}-f-\delta_{k}\right) d q .
\end{aligned}
$$

On the other hand, choosing $\phi=\psi\left(f_{k}-f+\delta_{k}\right)$ in (2.15) and (2.17) we infer

$$
\begin{aligned}
& \int_{N}|\nabla f|^{2} \psi d q-\int_{N}\left|\nabla f_{k}\right|^{2} \psi d q \leq 2 C_{2 A} \int_{N} \psi\left(f-f_{k}+\delta_{k}\right) d q \\
+ & \int_{N} \nabla f_{k} \cdot \nabla \psi\left(f_{k}-f+\delta_{k}\right) d q+\int_{N} \nabla f \cdot \nabla \psi\left(f_{k}-f+\delta_{k}\right) d q .
\end{aligned}
$$

We can use the local boundedness of $f_{k}$ in $W_{l o c}^{1,2}$ and Cauchy-Schwarz inequality to handle the second and third terms on the right hand side of (2.18) and (2.19). It follows that $\int_{N}\left|\nabla f_{k}\right|^{2} \psi d q \rightarrow \int_{N}|\nabla f|^{2} \psi d q$. Then

$$
\begin{aligned}
\int_{d(p, \cdot) \leq A}\left|\nabla f_{k}-\nabla f\right|^{2} d q \leq & \int_{N}\left|\nabla f_{k}-\nabla f\right|^{2} \psi d q=\int_{N}\left|\nabla f_{k}\right|^{2} \psi d q+\int_{N}|\nabla f|^{2} \psi d q \\
(2.20) & -2 \int_{N} \nabla f_{k} \cdot \nabla f \psi d q \rightarrow 0 .
\end{aligned}
$$

The derivation of (2.16) is similar to that of (2.6) in the proof of Lemma 2.2.

The case that $\Delta f_{k}$ are locally uniformly bounded from below in the weak sense is similar.

Next we extend the above results to the situation of a sequence of converging Riemannian manifolds. For simplicity of formulation we state a weaker version of the result.

Lemma 2.4. Let $\left(N_{k}, h_{k}, p_{k}\right)$ be a sequence of pointed complete Riemannian manifolds converging in $C^{1}$ to a pointed complete Riemannian manifold $(N, h, p)$. Let $f_{k} \in W_{l o c}^{1,2}\left(N_{k}\right) \cap L_{l o c}^{\infty}\left(N_{k}\right)$ which converges in $L_{l o c}^{\infty}$ and weakly in $W_{\text {loc }}^{1,2}$ to a function $f \in W_{\text {loc }}^{1,2}(N) \cap L_{\text {loc }}^{\infty}(N)$. Furthermore, we assume that the $\Delta f_{k}$ are locally uniformly bounded from above in the weak sense or locally uniformly bounded from below in the weak sense. Then $f_{k}$ converges strongly in $W_{\text {loc }}^{1,2}$ to $f$. Consequently, we have

$$
\int_{N_{k}}\left|\nabla f_{k}\right|^{2} \eta_{k} d q \rightarrow \int_{N}|\nabla f|^{2} \eta d q
$$

whenever $\eta \in L^{\infty}(N)$ and $\eta_{k} \in L^{\infty}\left(N_{k}\right)$ with support contained in $d_{h_{k}}\left(p_{k}, \cdot\right)<A$ for a fixed $A>0$, such that $\eta_{k}$ converges to $\eta$ in $L^{\infty}$.

Proof. The proof of Lemma 2.3 can easily be carried over. Note that the pointed convergence of $\left(N_{k}, h_{k}, p_{k}\right)$ to $(N, h, p)$ involves suitable embeddings from increasing domains of $N_{k}$ into $N$. Using these embeddings the functions $f_{k}$ can be transplanted to increasing domains of $N$. Then the difference between $f_{k}$ and $f$ can be measured. 


\section{Asymptotic limits of $\kappa$-SOlutions I}

Let $\tilde{g}(t),-\infty<t \leq 0$ be a $\kappa$-solution for some $\kappa>0$ on a manifold $M$. Recall [P1] that this means that $\tilde{g}(t)$ is a smooth solution of the Ricci flow

$$
\frac{\partial g}{\partial t}=-2 R i c
$$

on $M \times(-\infty, 0]$, such that for each $t$, the metric $\tilde{g}(t)$ is a complete nonflat metric of bounded and nonnegative curvature operator. Moreover, each $\tilde{g}(t)$ is $\kappa$-noncollapsed on all scales. (See [P1] for the definition of $\kappa$-noncollapsedness.) To understand the structures of $\tilde{g}(t)$, we analyse its rescaled asymptotical limits at the time infinity. One needs to use a blow-down rescaling, because the Ricci flow equation and nonnegative curvature imply that $\tilde{g}(t)$ expands as $t$ decreases.

Pick an arbitrary time $t_{0} \leq 0$. Then $g(\tau)=\tilde{g}\left(t_{0}-\tau\right), \tau \in[0, \infty)$, is a solution of the backward Ricci flow $(2.1)$ on $M \times[0, \infty)$. Obviously, $g$ retains the properties of $\tilde{g}$, namely $g$ is $\kappa$-noncollapsed on all scales and has a bounded and nonnegative curvature operator.

Next we choose an arbitary $p \in M$ as the $l$-base, i.e. the reference point for the $l$-function $l$ and the reduced volume $\tilde{V}$ (see [Y2, Definition 1]). We set

$$
\tilde{V}^{\infty}=\lim _{\tau \rightarrow \infty} \tilde{V}(\tau)
$$

By [Y2, Theorem 4.5], this limits exists and is finite.

For $a>0$, we set $g_{a}(\tau)=\frac{1}{a} g(a \tau)$. Choose a base point $\bar{q} \in M$ we then have a pointed solution of the backward Ricci flow $\left(g_{a}, M \times(0, \infty), \bar{q}\right)$. The following theorem is precisely [P1, Proposition 11.2].

Theorem 3.1. Let $\tau_{k} \rightarrow \infty$ be given. For each $\tau_{k}$, let $x\left(\tau_{k}\right)$ be a minimum point of $l(\cdot, \tau)$. Then the pointed flows $\left(g_{\tau_{k}}, M \times(0, \infty), x\left(\tau_{k}\right)\right)$ subconverge smoothly to pointed nonflat gradient shrinking solitons $\left(g_{\infty}, M_{\infty} \times(0, \infty), x_{\infty}\right)$.

In the remainder of this section, we establish the convergence part of Theorem 3.1 , and obtain some basic properties for the limit solutions. For convenience, we formulate the convergence part as a proposition.

Proposition 3.2. The pointed flows $\left(g_{\tau_{k}}, M \times(0, \infty), x\left(\tau_{k}\right)\right)$ subconverge smoothly to pointed solutions $\left(g_{\infty}, M_{\infty} \times(0, \infty), x_{\infty}\right)$ of the backward Ricci flow. The limits will be called asymptotical limits of $\tilde{g}$ or $g$.

Proof. The various quantities associated with $g_{\tau_{k}}$ will be indicated by the subscript $k$ or $g_{\tau_{k}}$, e.g. $l_{k}=l_{g_{\tau_{k}}}, \tilde{V}_{k}=\tilde{V}_{g_{\tau_{k}}}$ (the $l$-function and reduced volume associated with $\left.g_{\tau_{k}}\right)$, and $d_{k}=d_{g_{\tau_{k}}}$.

First note that by [Y2, Lemma 2.3] or [Y2, Lemma 3.2] the minimum points $x\left(\tau_{k}\right)$ exist. By [Y2, Lemma 3.1] and the scaling invariance of the l-function we have

$$
l_{k}\left(x\left(\tau_{k}\right), 1\right) \leq \frac{n}{2} .
$$

By this estimate and [Y2, Lemma 3.2] we infer that

$$
l_{k}(q, 1) \leq C_{2} d_{k}^{2}\left(x\left(\tau_{k}\right), q, 1\right)+n
$$

for all $q \in M$, where $C_{2}$ is a positive constant depending only on the dimension $n$. Then it follows from the Harnack inequality [Y2, (2.57)] that

$$
l_{k}(q, \tau) \leq \tau^{ \pm C}\left(C_{2}\left(d_{k}^{2}\left(x\left(\tau_{k}\right), q, 1\right)+n\right),\right.
$$


where $\pm=+$ if $\tau \geq 1, \pm=-$ if $\tau<1$, and $C$ is a positive constant depending only on $n$. Consequently, we obtain from [Y2, Theorem 2.18] and the nonnegativity of curvature operator the estimate

$$
|R m|_{k}(q, \tau) \leq C \tau^{-1 \pm C}\left(d_{k}^{2}\left(x\left(\tau_{k}\right), q, 1\right)+1\right) .
$$

By the $\kappa$-noncollapsing assumption and (3.6), we obtain for each $k$ and $\tau \in(0, \infty)$ local injectivity radius estimates for $g_{\tau_{k}}(\tau)$ which depend only on $d_{k}\left(x\left(\tau_{k}\right), \cdot, 1\right)$, $\tau$ (in terms of a positive lower bound and an upper bound of $\tau$ ), $\kappa$ and $n$. It is then straightforward to apply Gromov-Cheeger-Hamilton compactness $[\mathrm{H}]$ to obtain pointed smooth subconvergence of $\left(g_{\tau_{k}}, M \times(0, \infty), x\left(\tau_{k}\right)\right)$ to pointed solutions $\left(g_{\infty}, M_{\infty} \times(0, \infty), x_{\infty}\right)$ of the backward Ricci flow.

Let $\left(g_{\infty}, M_{\infty} \times(0, \infty), x_{\infty}\right)$ be an asymptotical limit. The corresponding converging subsequence will still be denoted by $g_{\tau_{k}}$. By [Y2, Theorem 2.18], the rescaling invariance and (3.5), the $l_{k}$ subconverge locally uniformly to locally Lipschitz functions $l_{\infty}$ on $M_{\infty} \times(0, \infty)$.

Definition 1. The limits $l_{\infty}$ will be called limit $l$-functions.

Lemma 3.3. Let $\left(g_{\infty}, M_{\infty} \times(0, \infty), x_{\infty}\right)$ be an asymptotical limit and $l_{\infty}$ a limit l-function. Then $l_{\infty}(\cdot, \tau)$ is locally Lipschitz for every $\tau$, and $l_{\infty}(q, \cdot)$ is locally Lipschitz for every q. Moreover, we have

$$
R_{\infty} \leq C \frac{l_{\infty}}{\tau}
$$

everywhere,

$$
\left|\nabla l_{\infty}\right| \leq C \frac{l_{\infty}}{\tau}
$$

almost everywhere in $M_{\infty}$ for each $\tau \in(0, \infty)$ (the $\nabla$ and the norm are those of $\left.g_{\infty}\right)$,

$$
\left|\frac{\partial l_{\infty}}{\partial \tau}\right| \leq C \frac{l_{\infty}}{\tau}
$$

almost everywhere in $(0, \infty)$ for each $q \in M_{\infty}$, and

$$
\left(\frac{\tau_{1}}{\tau_{2}}\right)^{C} \leq \frac{l_{\infty}\left(q, \tau_{2}\right)}{l_{\infty}\left(q, \tau_{1}\right)} \leq\left(\frac{\tau_{2}}{\tau_{1}}\right)^{C}
$$

for all $q \in M$ and $0<\tau_{1}<\tau_{2}$, where $R_{\infty}=R_{g_{\infty}}$ (the scalar curvature of $g_{\infty}$ ) and $C$ depends only on $n$.

Proof. These follow from the local uniform convergence of $l_{k}$ to $l_{\infty}$ and the corresponding properties of the $l$-function as given in [Y2, Theorem 2.18]. The estimates for the gradient and the $\tau$-derivative are obtained in terms of estimating the relevant Lipschitz constants.

Lemma 3.4. We have

$$
C_{1} \frac{d_{k}^{2}\left(x\left(\tau_{k}\right), q, \tau\right)}{\tau}-\frac{n}{2} \tau^{ \pm C}-1 \leq l_{k}(q, \tau) \leq C_{2} \frac{d_{k}^{2}\left(x\left(\tau_{k}\right), q, \tau\right)}{\tau}+n \tau^{ \pm C}
$$

for all $q \in M, \tau \in(0, \infty)$ and

$$
C_{1} \frac{d_{\infty}^{2}\left(x_{\infty}, q, \tau\right)}{\tau}-\frac{n}{2} \tau^{ \pm C}-1 \leq l_{\infty}(q, \tau) \leq C_{2} \frac{d_{\infty}^{2}\left(x_{\infty}, q, \tau\right)}{\tau}+n \tau^{ \pm C}
$$


for all $q \in M_{\infty}, \tau \in(0, \infty)$, where $d_{\infty}$ denotes the distance function of $g_{\infty}$, and the positive constant $C_{1}, C_{2}$ and $C$ depend only on the dimension $n$.

Proof. By the Harnack inequality [Y2, (2.57)] and (3.3) we deduce that

$$
l_{k}\left(x\left(\tau_{k}\right), \tau\right) \leq \frac{n}{2} \tau^{ \pm C} .
$$

Applying [Y2, Lemma 3.2] we then arrive at (3.11). Taking a limit leads to (3.12). (Note that a similar estimate is employed in the proof of [Y2, Theorem 3.3] in [Y2].)

Henceforth, we'll fix a limit $l$-function $l_{\infty}$ for each asymptotical limit. Obviously, the conclusions we derive are valid for each choice of the limit $l$-function.

Definition 2. Let $\left(g_{\infty}, M_{\infty} \times(0, \infty), x_{\infty}\right)$ be an asymptotical limit together with a limit $l$-function $l_{\infty}$. We define the asymptotical reduced volume to be

$$
\tilde{V}_{\infty}(\tau)=\int_{M_{\infty}} e^{-l_{\infty}} \tau^{-\frac{n}{2}} d q
$$

where $d q=d q_{g_{\infty}}$.

Lemma 3.5. $\tilde{V}_{k}(\tau)$ converges to $\tilde{V}_{\infty}(\tau)$ for each $\tau>0$ and hence there holds

$$
\tilde{V}_{\infty}(\tau)=\tilde{V}^{\infty}
$$

for each $\tau$.

Proof. Fix $\tau>0$. For each $A>0$ we have

$$
\tilde{V}_{k}(\tau)=\int_{d_{k}\left(x\left(\tau_{k}\right), \cdot, \tau\right) \leq A} e^{-l_{k}} \tau^{-\frac{n}{2}} d q_{k}+\int_{d_{k}\left(x\left(\tau_{k}\right), \cdot, \tau\right)>A} e^{-l_{k}} \tau^{-\frac{n}{2}} d q_{k}
$$

and

$$
\tilde{V}_{\infty}(\tau)=\int_{d_{\infty}\left(x_{\infty}, \cdot, \tau\right) \leq A} e^{-l_{\infty} \tau^{-\frac{n}{2}}} d q+\int_{d_{\infty}\left(x_{\infty}, \cdot, \tau\right)>A} e^{-l_{\infty} \tau^{-\frac{n}{2}}} d q .
$$

As in the proof of [Y2, Theorem 3.3] in [Y2], the lower bound in (3.11) and volume comparison imply

$$
\int_{d_{k}\left(x\left(\tau_{k}\right), \cdot, \tau\right)>A} e^{-l_{k}} \tau^{-\frac{n}{2}} d q_{k} \leq C e^{-c A}
$$

for suitable positive constants $C$ and $c$ depending only on $n$. Similarly, the lower bound in (3.12) and volume comparison imply

$$
\int_{d_{\infty}\left(x_{\infty}, \cdot, \tau\right)>A} e^{-l_{\infty} \tau^{-\frac{n}{2}}} d q \leq C e^{-c A} .
$$

(We can arrange the constants here to be the same as in (3.18).) By the pointed convergence of $\left(g_{\tau_{k}}, M \times(0, \infty), x\left(\tau_{k}\right)\right)$ to $\left(g_{\infty}, M_{\infty} \times(0, \infty), x_{\infty}\right)$ and the convergence of $l_{k}$ to $l_{\infty}$ we then infer $\lim \left|\tilde{V}_{k}(\tau)-\tilde{V}_{\infty}(\tau)\right| \leq 2 C e^{-c A}$. Since $A$ is arbitary, we conclude that $\lim \tilde{V}_{k}(\tau)=\tilde{V}_{\infty}(\tau)$. But $\tilde{V}_{k}(\tau)=\tilde{V}\left(\tau_{k} \tau\right) \rightarrow \tilde{V}^{\infty}$. Hence we arrive at (3.15). 


\section{Asymtotical limits of $\kappa$-SOlutions II}

The goal of this section is to establish the soliton characterization part of Theorem 3.1. We formulate it as a proposition. See [Y2] for the terminologies regarding gradient shrinking solitons used below.

Proposition 4.1. Each asymptotical limit $\left(g_{\infty}, M_{\infty} \times(0, \infty), x_{\infty}\right)$ is a pointed nonflat gradient shrinking soliton with time origin 0. Moreover, the limit l-functions $l_{\infty}$ are potential functions.

To prove this proposition, we need a few lemmas. We fix an asymptotical limit $\left(g_{\infty}, M_{\infty} \times(0, \infty), x_{\infty}\right)$ together with a limit $l$-function $l_{\infty}$. We have the corresponding converging subsequences $\left(g_{\tau_{k}}, M \times(0, \infty), x\left(\tau_{k}\right)\right)$ and $l_{k}$.

Lemma 4.2. For each $\tau>0, l_{k}(\cdot, \tau)$ converges strongly to $l_{\infty}(\cdot, \tau)$ in $W_{l o c}^{1,2}$. Consequently, we have

$$
\int_{M, g_{\tau_{k}}}\left|\nabla l_{k}\right|^{2} \phi_{k} d q \rightarrow \int_{M_{\infty}}\left|\nabla l_{\infty}\right|^{2} \phi d q
$$

whenever $\phi \in L^{\infty}\left(M_{\infty}\right)$ and $\phi_{k} \in L^{\infty}(M)$ such that the support of $\phi_{k}$ is contained in $d_{k}\left(x\left(\tau_{k}\right), \cdot \tau\right)<A$ for a fixed $A>0$, and that $\phi_{k}$ converges in $L^{\infty}$ to $\phi$.

Proof. By [Y2, Theorem 2.18] and (3.5), $l_{k}$ is a locally bounded sequence in $W_{l o c}^{1,2}$. Since it converges to $l_{\infty}$ in $L_{l o c}^{\infty}$, it also converges to $l_{\infty}$ weakly in $W_{l o c}^{1,2}$. Hence the conclusions of the lemma follow from Lemma 2.1, Lemma 2.4 and Lemma 3.4.

We also need the $L_{l o c}^{2}$ strong convergence of $\nabla l_{k}$ over the spacetime $M \times(0, \infty)$.

Lemma 4.3. The vector fields $\nabla l_{k}$ on $M \times(0, \infty)$ converge to the vector field $\nabla l_{\infty}$ on $M_{\infty} \times(0, \infty)$ strongly in $L_{l o c}^{2}$. Consequently, we have for arbitary $\tau_{2}>\tau_{1}>0$

$$
\int_{\tau_{1}}^{\tau_{2}} \int_{M, g_{\tau_{k}}}\left|\nabla l_{k}\right|^{2} \phi_{k} d q d \tau \rightarrow \int_{\tau_{1}}^{\tau_{2}} \int_{M_{\infty}}\left|\nabla l_{\infty}\right|^{2} \phi d q d \tau
$$

whenever $\phi \in L^{\infty}\left(M_{\infty} \times\left[\tau_{1}, \tau_{2}\right]\right)$ and $\phi_{k} \in L^{\infty}\left(M \times\left[\tau_{1}, \tau_{2}\right]\right)$, such that the support of $\phi_{k}$ is contained in $\left\{d_{k}\left(x\left(\tau_{k}\right), \cdot, 1\right)<A\right\} \times\left[\tau_{1}, \tau_{2}\right]$ for a fixed $A>0$, and that $\phi_{k}$ converges to $\phi$ in $L^{\infty}$.

Proof. By [Y2, Theorem 2.18] and (3.5), $l_{k}$ is a locally bounded sequence in $W_{l o c}^{1,2}$ over the spacetime. It follows that $l_{k}$ converges weakly in $W_{l o c}^{1,2}$ over the spacetime. Now we can argue as in the proof of Lemma 2.3, employing integrations over the spacetime with respect to the volume form $d q d \tau$. Note that the involved $\Delta$ and $\nabla$ are along the space with a time dependence on $\tau$.

Lemma 4.4. The equation

$$
\frac{\partial l_{\infty}}{\partial \tau}-\frac{R_{\infty}}{2}+\frac{\left|\nabla l_{\infty}\right|^{2}}{2}+\frac{l_{\infty}}{2 \tau}=0
$$

holds true almost everywhere on $M_{\infty} \times(0, \infty)$. The inequality

$$
\Delta l_{\infty}-\frac{\left|\nabla l_{\infty}\right|^{2}}{2}+\frac{R_{\infty}}{2}+\frac{l_{\infty}-n}{2 \tau} \leq 0
$$

holds true for each $\tau>0$ in the weak sense, i.e.

$$
\int_{M_{\infty}}\left\{-\nabla l_{\infty} \cdot \nabla \phi+\frac{1}{2}\left(-\left|\nabla l_{\infty}\right|^{2}+R_{\infty}+\frac{l_{\infty}-n}{\tau}\right) \phi\right\} d q \leq 0
$$


for all nonnegative Lipschitz functions $\phi$ with compact support. Finally, the inequality

$$
\frac{\partial l_{\infty}}{\partial \tau}-\Delta l_{\infty}+\left|\nabla l_{\infty}\right|^{2}-R_{\infty}+\frac{n}{2 \tau} \geq 0
$$

holds true on $M_{\infty} \times(0, \infty)$ when $\Delta$ is interpreted in the weak sense, i.e.

$$
Q_{\tau_{1}, \tau_{2}}(\phi) \geq 0
$$

for arbitray $\tau_{2}>\tau_{1}>0$ and nonnegative Lipschitz functions $\phi$ on $M_{\infty} \times\left[\tau_{1}, \tau_{2}\right]$ with compact support, where

$$
Q_{\tau_{1}, \tau_{2}}(\phi)=\int_{\tau_{1}}^{\tau_{2}} \int_{M_{\infty}}\left\{\nabla l_{\infty} \cdot \nabla \phi+\left(\frac{\partial l_{\infty}}{\partial \tau}+\left|\nabla l_{\infty}\right|^{2}-R_{\infty}+\frac{n}{2 \tau}\right) \phi\right\} d q d \tau .
$$

Proof. First consider a fixed $\tau>0$. Let $\phi$ be a nonnegative smooth function on $M_{\infty}$ with compact support. Let $\phi_{k}$ be a sequence of nonnegative smooth functions on $M$ such that the support of $\phi_{k}$ is contained in $d_{k}\left(x\left(\tau_{k}\right), \cdot \tau\right)<A$ for a fixed $A>0$, and that $\phi_{k}$ converges smoothly to $\phi$. By [Y2, Theorem 2.23] we have for each $k$

$$
\int_{M, g_{\tau_{k}}}\left\{-\nabla l_{k} \cdot \nabla \phi_{k}+\left(-\frac{\left|\nabla l_{k}\right|^{2}}{2}+\frac{R_{k}}{2}+\frac{l_{k}-n}{2 \tau}\right) \phi_{k}\right\} d q \leq 0 .
$$

By Lemma 4.2 and the pointed convergence of $\left(g_{\tau_{k}}, M \times(0, \infty), x\left(\tau_{k}\right)\right)$ to $\left(g_{\infty}, M_{\infty} \times\right.$ $\left.(0, \infty), x_{\infty}\right)$ we can take the limit in $(4.9)$ to arrive at $(4.5)$ with the above $\phi$. Then it follows that (4.5) also holds true for nonnegative Lipschitz or Sobolev functions $\phi$ with compact support.

Next consider $\tau_{2}>\tau_{1}>0$ and a smooth nonnegative function $\phi$ on $M_{\infty} \times\left[\tau_{1}, \tau_{2}\right]$ with compact support. Let $\phi_{k}$ be a sequence of smooth functions on $M \times\left[\tau_{1}, \tau_{2}\right]$ such that the support of $\phi_{k}$ is contained in $d_{k}\left(x\left(\tau_{k}\right), \cdot, 1\right)<A$ for a fixed $A>0$, and that $\phi_{k}$ converges smoothly to $\phi$. By [Y2, Theorem 2.23] we have

$$
\int_{\tau_{1}}^{\tau_{2}} \int_{M, g_{\tau_{k}}}\left\{\nabla l_{k} \cdot \nabla \phi_{k}+\left(\frac{\partial l_{k}}{\partial \tau}+\left|\nabla l_{k}\right|^{2}-R_{k}+\frac{n}{2 \tau}\right) \phi_{k}\right\} d q d \tau \geq 0 .
$$

Since $\frac{\partial}{\partial \tau} d q=R_{k} d q$ we can rewrite this equation as follows:

$$
\begin{aligned}
& \left.\int_{M, g_{\tau_{k}}} l_{k} \phi_{k} d q\right|_{\tau_{2}}-\left.\int_{M, g_{\tau_{k}}} l_{k} \phi_{k} d q\right|_{\tau_{1}} \\
& +\int_{\tau_{1}}^{\tau_{2}} \int_{M, g_{\tau_{k}}}\left\{\nabla l_{k} \cdot \nabla \phi_{k}+\left(-l_{k} \frac{\partial \phi_{k}}{\partial \tau}+\left|\nabla l_{k}\right|^{2}-2 R_{k}+\frac{n}{2 \tau}\right) \phi_{k}\right\} d q d \tau \geq 0 .
\end{aligned}
$$

By Lemma 4.3, the local uniform convergence of $l_{k}$ to $l_{\infty}$ and the pointed convergence of $\left(g_{\tau_{k}}, M \times(0, \infty), x\left(\tau_{k}\right)\right)$ we can take the limit in (4.11) to arrive at

$$
\begin{aligned}
& \left.\int_{M_{\infty}} l_{\infty} \phi d q\right|_{\tau_{2}}-\left.\int_{M_{\infty}} l_{\infty} \phi d q\right|_{\tau_{1}} \\
& +\int_{\tau_{1}}^{\tau_{2}} \int_{M_{\infty}}\left\{\nabla l_{\infty} \cdot \nabla \phi+\left(-l_{\infty} \frac{\partial \phi}{\partial \tau}+\left|\nabla l_{\infty}\right|^{2}-2 R_{\infty}+\frac{n}{2 \tau}\right) \phi\right\} d q d \tau \geq 0
\end{aligned}
$$

which is equivalent to (4.7) for the above $\phi$. Then (4.7) also holds true for nonnegative Lipschitz or Sobolev $\phi$ with compact support. 
By [Y2, Lemma 2.22] and [Y2, Proposition 2.16] we have

$$
\frac{\partial l_{k}}{\partial \tau}-\frac{R_{k}}{2}+\frac{\left|\nabla l_{k}\right|^{2}}{2}+\frac{l_{k}}{2 \tau}=0
$$

almost everywhere on $M \times(0, \infty)$. We turn this equation into an integral equation in the same fashion as (4.11). Taking the limit we then arrive at a corresponding integral equation for $l_{\infty}$, which implies that (4.3) holds true almost everywhere.

Proof of Proposition 4.1. Fix an asymptotical limit $\left(g_{\infty}, M_{\infty} \times(0, \infty), x_{\infty}\right)$ together with a limit $l$-function $l_{\infty}$ as above. By (4.7), Lemma 3.4 and the arguments in the proof of [Y2, Theorem 3.3] in [Y2] we infer that $Q_{\tau_{1}, \tau_{2}}(\phi) \geq 0$ holds true for nonnegative admissible locally Lipschitz $\phi$ on $M_{\infty} \times\left[\tau_{1}, \tau_{2}\right]$, where "admissible" means that $\phi \leq C \tau^{-\frac{n}{2}} e^{-l_{\infty}}$ and $|\nabla \phi| \leq C \tau^{-\frac{n+1}{2}} \sqrt{l_{\infty}} e^{-l_{\infty}}$ for all $q \in M_{\infty}$ and $\tau \in\left[\tau_{1}, \tau_{2}\right]$ and some positive constant $C$ depending on $\phi$. On the other hand, we can argue as in the proof of [Y2, Lemma 4.6] in [Y2] to deduce

$$
\tilde{V}_{\infty}\left(\tau_{2}\right)-\tilde{V}_{\infty}\left(\tau_{1}\right)=-\int_{\tau_{1}}^{\tau_{2}} \int_{M_{\infty}}\left(\frac{\partial l_{\infty}}{\partial \tau}-R_{\infty}+\frac{n}{2 \tau}\right) e^{-l_{\infty}} \tau^{-\frac{n}{2}} d q d \tau
$$

By Lemma 3.5 we then infer that $Q\left(\tau^{-\frac{n}{2}} e^{-l_{\infty}}\right)=0$. As in the proof of [Y2, Theorem 4.9] in [Y2], we then deduce that $Q_{\tau_{1}, \tau_{2}}(\phi)=0$ for all admissible locally Lipschitz $\phi$. By basic regularity theory for parabolic equations we derive that $l_{\infty}$ is smooth and the equation

$$
\frac{\partial l_{\infty}}{\partial \tau}-\Delta l_{\infty}+\left|\nabla l_{\infty}\right|^{2}-R_{\infty}+\frac{n}{2 \tau}=0
$$

holds true everywhere. By Lemma 4.4, equation (4.3) also holds true everywhere. Taking the difference between (4.3) and (4.15) we then arrive at the equation

$$
\Delta l_{\infty}-\frac{\left|\nabla l_{\infty}\right|^{2}}{2}+\frac{R_{\infty}}{2}+\frac{l_{\infty}-n}{2 \tau}=0
$$

By (4.15), (4.16) and [Y2, Lemma 4.8] we finally conclude that $g_{\infty}$ is a gradient shrinking soliton with time origin 0 and potential function $l_{\infty}$.

Now we show that $g_{\infty}$ is nonflat. Assume that $g_{\infty}$ is flat. Then the soliton equation $[\mathrm{Y} 2,(4.16)]$ implies

$$
\nabla_{g_{\infty}}^{2} l_{\infty}=\frac{1}{2 \tau} g_{\infty}
$$

It follows that $l_{\infty}(\cdot, 1)$ is a strictly convex function with a unique minimum point (by the construction it has a minimum point $x_{\infty}$ ). By Lemma 3.4, its level sets are compact. Employing the gradient flow of $l_{\infty}(\cdot, 1)$ one readily shows that $M_{\infty}$ is diffeomorphic to $\mathbf{R}^{n}$ and then each $\left(M_{\infty}, g_{\infty}(\tau)\right)$ is isometric to $\mathbf{R}^{n}$. Next we observe that (4.17) implies that $\Delta_{g_{\infty}} l_{\infty}=\frac{n}{2 \tau}$, which together with (4.16) leads to

$$
\left|\nabla l_{\infty}\right|^{2}=\frac{l_{\infty}}{\tau}
$$

For a fixed $\tau$, we identify $\left(M_{\infty}, g_{\infty}\right)$ with $\mathbf{R}^{n}$ via an isometry. Let $x$ denote the coordinates on $\mathbf{R}^{n}$. Then (4.17) implies that

$$
\nabla l_{\infty}(x, \tau)=\nabla \frac{|x|^{2}}{4 \tau}+v(\tau)
$$


for a constant vector $v(\tau)$. Hence

$$
l_{\infty}(x, \tau)=\frac{|x|^{2}}{4 \tau}+v(\tau) \cdot x+c(\tau)
$$

for a constant $c(\tau)$. A simple calculation using (4.18), (4.19) and (4.20) yields $c(\tau)=\tau|v|^{2}$. It follows that $l_{\infty}(x, \tau)=\frac{|x+2 \tau v|^{2}}{4 \tau}$ and hence $\tilde{V}_{\infty}(\tau)=(4 \pi)^{\frac{n}{2}}$ for each $\tau$. But $\tilde{V}_{\infty}(\tau)=\tilde{V}^{\infty}<(4 \pi)^{\frac{n}{2}}$ by [Y2, Theorem 4.4] and [Y2, Theorem 4.5]. Thus we arrive at a contradiction.

\section{ReFERENCES}

[H] R. Hamilton, A compactness property for solutions of the Ricci flow, Amer. J. Math. 117 (1995), 545-572. MR1333936 (96c:53056)

[P1] G. Perelman, The entropy formula for the Ricci flow and its geometric applications, arXiv:math.DG/0211159.

[P2] G. Perelman, Ricci flow with surgeries on three-manifolds, arXiv:math.DG/0303109.

[Y1] R. Ye, On the l-function and the reduced volume of Perelman.

[Y2] R. Ye, On the l-function and the reduced volume of Perelman I, this issue.

Department of Mathematics, University of California, Santa Barbara, California 93106

E-mail address: yer@math.ucsb.edu 